\title{
Corela
}

Cognition, représentation, langage

13-1 | 2015

Vol. $13, \mathrm{n}^{\circ} 1$

\section{Le passif en français et les lacunes distributionnelles des verbes}

Proposition d'analyse sémantique invariante

\section{Pierre Jalenques}

\section{(2) OpenEdition}

\section{Journals}

Édition électronique

URL : http://journals.openedition.org/corela/4015

DOI : $10.4000 /$ corela.4015

ISSN : 1638-573X

Éditeur

Cercle linguistique du Centre et de I'Ouest - CerLICO

Référence électronique

Pierre Jalenques, «Le passif en français et les lacunes distributionnelles des verbes », Corela [En ligne], 13-1 | 2015, mis en ligne le, consulté le 19 avril 2019. URL : http://journals.openedition.org/ corela/4015; DOI : 10.4000/corela.4015

Ce document a été généré automatiquement le 19 avril 2019

\section{cc)(1)(2)}

Corela - cognition, représentation, langage est mis à disposition selon les termes de la licence Creative Commons Attribution - Pas d'Utilisation Commerciale - Partage dans les Mêmes Conditions 4.0 International. 


\section{Le passif en français et les lacunes distributionnelles des verbes}

Proposition d'analyse sémantique invariante

Pierre Jalenques

\section{Introduction}

\subsection{Objet d'étude}

1 Nous reprenons ici l'étude de l'objet linguistique appelé, selon les auteurs, "passif périphrastique » (cf. Gaatone, 1998; Helland, 2002) ou simplement « passif» (cf. Milner, 1985 ; Raineri 2010). D’un point de vue purement descriptif, on s'accorde généralement à reconnaître dans cet objet linguistique la conjonction des caractéristiques suivantes :

- -le second argument du verbe (noté Y) est en position de sujet syntaxique ;

-le premier argument (noté $\mathrm{X}$ ) occupe une position syntaxiquement facultative ;

-le verbe apparaît sous la forme de participe passé ;

-l'élément ${ }^{1}$ être est intercalé entre le sujet et la participe passé.

2 Cet objet linguistique est souvent présenté en parallèle avec la configuration dite active. Il s'agit dans le présent article d'une simple commodité descriptive, sans préjuger d'une quelconque relation entre les deux configurations.

(1) tous [X] connaissent cette histoire $[Y]$

(2) cette histoire [Y] est connue de tous [X]

3 Le passif a fait l'objet de multiples analyses sous de multiples point de vue (syntaxique, grammatical, sémantique, pragmatique (pour un panorama général, cf. Gaatone (1998) ; Helland (2002); Raineri (2010)). Nous nous proposons de reprendre son étude à partir de données généralement absentes ou marginalisées dans son analyse, à savoir les lacunes distributionnelles par rapport à l'actif. Considérons un autre emploi du verbe connaître :

(3) notre époque connaît de grands bouleversements

4 Contrairement à l'emploi (1), l'emploi (3) donne lieu à une séquence difficilement acceptable au passif: 
(1') cette histoire est connue de tous

(3') ?? de grands bouleversements sont connus de notre époque

Prenons encore les deux emplois suivants du verbe visiter:

(4)a. durant le week-end, des cambrioleurs ont visité la bijouterie, il ne reste plus rien.

(4)b. Paul a visité la Chine cet été

6 Contrairement à l'emploi (4a), l'emploi (4b) donne lieu à une séquence difficilement acceptable au passif :

(5)a. durant le week-end, la bijouterie a été visitée par des cambrioleurs, il ne reste plus rien.

(5)b. ?? la Chine a été visitée par Paul cet été

7 Donc, il ne suffit pas qu'un verbe du français ait un emploi en construction directe pour que cet emploi donne automatiquement lieu à un emploi au passif. Ces faits sont bien connus et de nombreuses classes d'emplois de verbes concernés par cette situation ont été recensées dans la littérature ${ }^{2}$.

\subsection{Une double problématique}

8 Toute la question est de savoir si les données illustrées en (3') et b) constituent autant d'exceptions à la passivabilité, c'est-à-dire constituent des idiosyncrasies lexicales, ou bien si elles relèvent au contraire d'un principe régulier.

9 Notons d'emblée que les verbes concernés par cette situation sont généralement polysémiques, comme les verbes connaître ou visiter dans les exemples ci-dessus. Il apparaît ainsi que l'impassivabilité ne concerne habituellement qu'une partie des emplois de ces verbes, argument général qui laisserait penser à des idiosyncrasies lexicales. En tout cas, l'impassivabilité ne concerne pas une liste de verbes mais une liste d'emplois de verbes ${ }^{3}$. Nous insistons sur ce point.

10 Si l'on fait l'hypothèse que ces lacunes distributionnelles correspondent à des idiosyncrasies lexicales, leur existence est sans conséquence sur l'analyse du passif. Précisément, parce que c'est l'hypothèse la plus courante, ce type de données n'est généralement pas pris en compte dans l'analyse du passif. Mais, si ces lacunes distributionnelles relèvent d'un principe régulier, alors la question du lien entre ce principe régulier et les propriétés du passif se pose, puisque ces lacunes s'observent au passif et non à l'actif. Nous avons donc deux questions à aborder :

- est-ce que ces lacunes distributionnelles peuvent être ramenées à un principe régulier?

- si régularité il y a, peut-on tirer de celle-ci une hypothèse sur les propriétés du passif luimême?

11 Nous répondrons positivement à chacune de ces deux questions. Dans la première partie de l'article, nous proposerons une nouvelle hypothèse, de nature sémantique, permettant de régulariser les lacunes distributionnelles au passif. Dans la deuxième partie de l'article nous verrons que cette hypothèse, de par son contenu sémantique, conforte l'analyse lexicale du passif, c'est-à-dire l'hypothèse selon laquelle l'élément être au passif n'est pas une simple marque formelle du passif, en elle-même vide de sens, mais correspond au contraire au verbe être lexicalement plein.

Plus précisément, dans cette deuxième partie de l'article, nous défendrons la nécessité de combiner des observations relevant de dimensions du passif généralement traitées de 
façon isolée ; il s'agit de données relevant de la syntaxe, de la sémantique grammaticale et de la sémantique lexicale. Nous verrons que leur combinaison permet de mettre en évidence une convergence de faits à l'appui de l'analyse lexicale du passif. Cette analyse nous permettra enfin de repréciser la relation entre passivabilité et transitivité, ainsi que le statut du complément d'agent au passif.

\subsection{Positionnement par rapport à des débats non abordés ici}

13 Avant d'aborder la première partie, précisons encore quelques points généraux sur le passif. On le sait, le passif a fait et fait encore l'objet de nombreux débats entre cadres théoriques et parfois à l'intérieur des cadres théoriques. Nous ne saurions les reprendre tous ici. Ceci dit, pour limiter autant que possible les malentendus éventuels dans la suite de l'article, précisons trois d'entre eux.

Le premier débat concerne l'unité même du phénomène appelé "passif ». Dans de nombreuses langues, mais pas toutes, on identifie une ou des constructions ${ }^{4}$ particulières que l'on étiquette " passif ». Une des questions qui a animé l'étude du passif était alors de savoir si l'on pouvait ramener toutes ces constructions particulières à un type général, c'est-à-dire à une caractérisation invariante qui subsume leur diversité (cf. D. Perlmutter \& P. Postal, 1977).

A l'encontre des approches universalistes, plusieurs études typologiques du passif ont montré l'extrême diversité des propriétés des constructions dites passives. Ainsi, A. Siewierska (1985) affirme dès le début de sonouvrage: "The analysis of the various constructions referred to in the literature as passive leads to the conclusion that there is not even one single property which all these constructions have in common » (p. 1). Plus récemment, W. Croft (2001: 283) aboutit à la même conclusion, affirmant que les constructions (passives) sont des objets singuliers, spécifiques à chaque langue ("Constructions are language-specific »).

Ainsi, nous considérons l'objet dont nous parlons ici comme une construction spécifique au français, telle que nous l'avons définie au début de l'introduction. Nous ne prétendons en aucune façon que les conclusions auxquelles nous aboutissons dans cette étude seraient transposables telles quelles à des constructions également étiquetées "passif » dans d'autres langues ou bien à d'autres constructions en français parfois étiquetées «passif» (comme le passif pronominal).

Un second débat dans l'étude du passif concerne le statut du complément d'agent. On peut distinguer deux grandes tendances opposées. Dans la première tendance, les auteurs affirment que le passif correspond à la perte d'un argument pour le verbe, en l'occurrence la perte de son premier argument (celui qui correspond au complément d'agent $)^{5}$; nous aurions donc une réduction de la valence du verbe. Dans cette hypothèse, le complément d'agent ne correspondrait pas à un argument du verbe, mais aurait un rôle proche de celui de complément circonstanciel. Il s'agit de l'analyse classiquement défendue en grammaire générative (cf. Helland, 2002). En dehors de ce cadre, le même point de vue est défendu dans Desclès \& Guentchéva (1993), Lamiroy (2000).

18 Dans la seconde tendance sur le statut du complément d'agent, les auteurs considèrent que la valence du verbe ne change pas au passif; le complément d'agent est bien un argument du verbe (son premier argument). Cependant, par différence avec l'actif, ce premier argument est mis à l'écart de la relation prédicative, marginalisé, d'une façon ou 
d'une autre. En tout cas, il ne disparaît pas, mais change de statut syntaxico-sémantique. C'est le point de vue défendu par exemple dans Gaatone (1998), C. Muller (2002). Notre analyse du passif se situe dans cette seconde tendance en ce qui concerne la valence du verbe. Nous considérons donc que le complément d'agent est bien un argument du verbe. Nous reviendrons sur le statut sémantique de ce complément à la fin de l'article.

Un troisième débat concerne le statut du participe passé au passif. Un certain nombre d'approches considèrent que cet objet linguistique a des propriétés spécifiques qui le distinguent du participe passé aux temps composés de l'actif (passé composé, etc.) et dans la construction pronominale. Certains auteurs parlent ainsi de "participe passé passif». Cette analyse revient à considérer que les participes passés à l'actif et au passif sont des homonymes (cf. Langacker, $1991: 201$; Helland, 2002: 45). Cette thèse homonymique du participe passé pose de nombreux problèmes; mais la thèse inverse admettant que le participe passé est le même objet linguistique à l'actif et au passif pose également des problèmes de cohérence à notre connaissance non résolus. Ce débat excède donc largement les limites de notre article. Nous ne prendrons donc pas position sur ce point. Nous parlerons simplement de "participe passé », au passif, sans préjuger de ses liens éventuels avec le participe passé à l'actif.

Dans la mesure où les faits que nous reprenons et que nous nous proposons d'articuler ont été mis au jour dans des cadres théoriques variés, l'analyse que nous développons est transversale à ces différentes approches. Aussi, l'enjeu de cet article est avant tout descriptif. Ceci dit, sur le plan épistémologique, dans le fait de combiner des observations relevant de différents domaines de la langue (syntaxique, sémantique, grammatical), notre démarche rejoint le programme de recherche initié par Antoine Culioli au sein de la Théorie des opérations prédicatives et énonciatives; en particulier lorsqu'il affirme à propos des différentes dimensions des langues que « l'on peut fournir une théorie unifiée intégrant des phénomènes répartis, à l'heure actuelle, dans des secteurs séparés (Culioli, [1982] 1999 : 96).

\section{Mise au jour d'une régularité sémantique dans les lacunes distributionnelles au passif}

Dans un premier temps, en 2.1., nous reprendrons les principales propositions de principe régulier pour ces lacunes distributionnelles et rappellerons leurs limites. Dans les sections suivantes, 2.2. à 2.5., nous développerons une nouvelle hypothèse, permettant de ramener ces différentes lacunes distributionnelles à une même propriété invariante. Cette propriété est de nature sémantique. Nous repartirons en 2.2. de l'analyse que nous avions développée dans Jalenques (2010) concernant les lacunes distributionnelles avec les procès dynamiques transitionnels ${ }^{6}$. En effet, ces données mettent en relief un aspect crucial des contraintes imposées par le passif. Ensuite, nous montrerons que l'analyse peut être étendue aux autres types de procès, dynamiques non transitionnels et statifs.

\subsection{Rappel des hypothèses précédentes sur les lacunes distributionnelles}

Depuis les années 70, plusieurs hypothèses ont été proposées pour tenter de ramener tout ou partie de ces lacunes distributionnelles à un ou plusieurs principe(s) régulier(s). D. 
Gaatone (1998) les a reprises une à une et a relevé pour chacune d'elle des contreexemples, parfois nombreux; nous ne reprendrons pas leur discussion en détail et renvoyons pour cela à l'auteur.

Citons tout d'abord l'hypothèse selon laquelle les emplois difficilement passivables correspondraient à des emplois figés, idiomatiques. En effet, une locution verbale comme prendre corps (exemple repris à Gaatone, p. 153) n'est guère passivable :

(6)a. Ce projet ambitieux a enfin pris corps

(6)b. * corps a enfin été pris (par ce projet ambitieux)

Tout le problème est que l'on trouve par ailleurs de nombreuses locutions verbales parfaitement passivables, comme par exemple faire abstraction de :

(7) abstraction a été faite de l'île Bahreïn, considérée comme iranienne. ${ }^{7}$

En particulier, Gaatone (1998) relève un certain nombre de séquences ayant une double lecture sémantique, compositionnelle et figée, comme briser la glace ou couper la poire en deux. Celles-ci sont passivables aussi bien dans leur emploi figé que compositionnel :

(8)a. au bord du lac, la glace a été brisée en plusieurs endroits par les pêcheurs

(8)b. après une heure de discussion, la glace a enfin été brisée entre les négociateurs

(9)a. dans cette recette de cuisine, la poire est coupée en deux dans le sens

horizontal

(9)b. après des heures de tractations, finalement, la poire a été coupée en deux

On pourrait multiplier les exemples (justice a été rendue / les points ont été mis sur les « $i$ ", etc.). Il n'y a donc pas de corrélation entre le caractère idiomatique d'une expression verbale et son degré de passivabilité.

Une autre hypothèse classique concerne les emplois figurés des verbes. A la suite de Gross (1975: 85), C. Leclère (1993) observe que lorsqu'un verbe polysémique a des emplois figurés, ceux-ci semblent plus difficilement passivables. L'auteur observe en particulier que l'emploi figuré est difficilement passivable lorsqu'il correspond à un sens métaphorique. Ainsi, Leclère relève (p. 8) que le verbe collectionner est passivable dans son sens premier illustré en a) et a) mais pas dans son sens métaphorique illustré en b) et b) :

(10)a. beaucoup de gens collectionnent les porte-clefs

(10)b. beaucoup de gens collectionnent les ennuis

(11)a. les porte-clefs sont collectionnés par beaucoup de gens

(11)b. ?? les ennuis sont collectionnés par beaucoup de gens

Là encore, Gaatone (1998) relève de nombreux contre-exemples avec des verbes parfaitement passivables dans leurs emplois « figurés " tout autant que dans leurs sens concrets ou premiers ; ainsi est-ce le cas des exemples suivants où le sens figuré du verbe apparaît dans la séquence $b$ :

(12)a. un homme a été heurté par une voiture sur le boulevard Saint-Michel

(12)b. Paul a été heurté par les propos du directeur

(13)a. la ville de Fukushima a été touchée par un nouveau séisme

(13)àb. le peuple japonais a été touché par l'élan de solidarité international

En dehors de ces deux hypothèses traditionnelles, nous pouvons ramener l'essentiel des discussions sur les lacunes distributionnelles au problème de la transitivité, définie sur les plans syntaxique ou / et sémantique. De nombreux auteurs envisagent actuellement la transitivité comme une notion gradable, associée à l'idée qu'il y aurait des emplois de verbe dont la transitivité est prototypique et des emplois moins prototypiques. Pour un panorama sur la transitivité sémantique, je renvoie à J. François (1998). Sur le plan syntaxique, la notion de transitivité est elle-même associée à la notion de complément 
d'objet direct, elle aussi pouvant être envisagée de façon gradable (il y a des C.O.D. plus ou moins typiques) (cf. L. Mélis, 2002). directe seraient difficilement passivables parce que faiblement transitifs (sur le plan syntaxique et / ou sémantique). Passons sur les risques de raisonnement circulaire dans le cas de la transitivité définie syntaxiquement, puisque, on le sait, la passivabilité est justement un des critères définissant le « vrai » COD et donc la transitivité syntaxique.

31 Mais peu importe car de toute façon le degré de passivabilité d'un emploi de verbe avec un complément direct n'est pas proportionnel au degré de transitivité de cet emploi. Ainsi, on trouve des emplois proches du prototype de transitivité (syntaxique et sémantique) et pourtant difficilement passivables. Prenons l'exemple du verbe regarder, dans l'emploi suivant :

(14) Marie a regardé la télé toute la soirée

Cet emploi du verbe regarder correspond à un procès dynamique ${ }^{8}$ agentif avec un sujet animé humain, donc proche du prototype sémantique de transitivité. De plus, syntaxiquement, le verbe se construit ici obligatoirement avec un complément d'objet direct. Cet emploi de regarder est pourtant difficilement passivable :

(15) ?? la télé a été regardée par Marie toute la soirée.

Symétriquement, on trouve des verbes essentiellement intransitifs comme vivre, pleurer ou courir, donc éloignés du prototype de transitivité, et pourtant parfaitement passivables dans certains de leurs emplois en construction directe ${ }^{9}$ :

(16)a. cet incident a été mal vécu par les passagers.

(16)b. Mandela mort il y a un mois a été pleuré par le monde entier.

(16)c. Au-delà de toute considération partisane, le décès du sénateur du Massachusetts Ted Kennedy a été pleuré par l'ensemble de la classe politique américaine.

(16)d. Chaque étape a été courue par un peloton d'une $50^{\text {aine }}$ de collégiens, lycéens et professeurs.

(16)e. Ça y est, c'est fait, une première finale en coupe de France a été courue par un pilote Limousin.

L'hypothèse bien connue de Milner (1986) n'est qu'un cas particulier de ce problème général de la relation supposée entre degré de passivabilité et degré de transitivité. L'auteur suppose que les emplois non passivables seraient les emplois des verbes correspondant à des procès non agentifs et statifs ${ }^{10}$. Cela se vérifie en effet pour un certains nombres de verbes, comme le verbe mesurer:

(17)a. l'âge de l'univers a été mesuré avec précision.

(17)b. * $1 \mathrm{~m} 76$ sont mesurés par Paul.

Mais, face à cette hypothèse, Gaatone (1998) relève de nombreux emplois de verbes polysémiques qui sont tout aussi passivables dans leurs emplois non agentifs et statifs que dans leurs emplois correspondant à un procès dynamique, comme le verbe séparer :

(18)a. les deux jumeaux ont été séparés à la naissance.

(18)b. les deux pièces sont séparées par une cloison

Notons que dans ces derniers exemples et, l'élément être du passif est tantôt au passé composé tantôt au présent. Certes, cette variation peut avoir un effet sur le type de procès associé au participe passé (le passé composé pouvant favoriser une interprétation dynamique du procès, le présent pouvant favoriser une interprétation stative). Mais, cette variation aspectuo-temporelle est sans conséquence ici puisque nous venons de voir 
que le passif marche aussi bien avec les procès dynamiques que les procès statifs. En l'occurrence, on observe que dans b) et b) le temps est le même (présent). Pourtant, seul b) est acceptable. Inversement, en , être est au passé composé et la séquence est néanmoins difficilement acceptable au passif. Le temps du verbe n'intervient donc pas dans le degré d'acceptabilité d'un emploi au passif. C'est pourquoi dans les exemples que nous donnons l'élément être peut se trouver indifféremment au passé composé, au présent ou à un autre temps.

L'ensemble de ces observations amène Gaatone à invalider les différentes propositions de propriété régulière pour décrire ces lacunes distributionnelles. Cela le conduit à considérer que "[l']étude minutieuse des données révèle un comportement hautement idiosyncrasique des verbes par rapport au passif» Gaatone (1998: 66); en d'autres termes, l'auteur affirme qu'il n'y a pas de principe régulier permettant de rendre compte de l'ensemble de ces lacunes distributionnelles.

\subsection{Résultats de Jalenques (2010) concernant les procès dynamiques transitionnels}

La plupart des emplois impassivables qui ont été recensés dans la littérature consacrée aux lacunes distributionnelles du passif correspondent à des emplois statifs. Or, comme nous l'avons souligné dans Jalenques (2010), on trouve aussi de nombreux emplois de verbe correspondant à un procès dynamique difficilement passivables. Nous nous étions intéressé en particulier aux emplois de verbes correspondant à un procès transitionnel. Considérons les emplois suivants des verbes hisser et monter:

(19)a. les enfants ont hissé le drapeau à toute vitesse

(19b. le drapeau a été hissé à toute vitesse par les enfants

(20)a. les enfants ont monté les escaliers en courant

(20b. ?? les escaliers ont été montés en courant par les enfants

Ces deux emplois de verbes sont sémantiquement proches, évoquant tous les deux un déplacement vers le haut. Cependant il existe une différence cruciale entre ces deux emplois, elle concerne ce que l'auteur désigne comme la portée sémantique du procès : en le déplacement vers le haut concerne le drapeau, c'est-à-dire le second argument du verbe, $\mathrm{Y}$; au contraire, en, le déplacement vers le haut concerne les enfants, c'est-à-dire le premier argument du verbe, $\mathrm{X}$, ce que l'on peut représenter dans la figure suivante :

\section{a. les enfants ont hissé le drapeau à toute vitesse}

a. les enfants ont monté les escaliers en courant

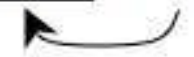

Il apparaît ainsi de façon générale que lorsque la transition du procès concerne le second argument, l'emploi est passivable ; lorsqu'elle concerne le premier argument, l'emploi du verbe est difficilement passivable. Prenons un autre emploi de monter, sémantiquement proche de , exprimant également un déplacement vers le haut; mais cette fois-ci la transition porte sur Y (le déplacement vers le haut concerne Y). Nous constatons alors que l'emploi du verbe est passivable :

(21)a. on a monté les valises dans votre chambre

(21)b. les valises ont été montées dans votre chambre 
41 Alors que le passif est souvent présenté comme une construction symétrique de l'actif, ces données montrent au contraire une dissymétrie entre les deux constructions : à l'actif, le procès peut aussi bien porter sur le premier que sur le second argument (cf. a) et a) ; au passif, la portée sémantique du procès est contrainte sur le second argument uniquement.

42 Reprenons l'exemple. Il se trouve qu'en combinaison avec le nom escalier, le verbe monter admet une autre interprétation ${ }^{11}$, proche de "assembler», comme dans l'exemple suivant :

(22) les ouvriers ont monté l'escalier en deux heures, il leur reste juste à installer la rampe.

43 Or, dans cet emploi, la transition concerne $\mathrm{Y}$, contrairement à a) où elle concernait $\mathrm{X}$. Lorsque l'on regarde les données attestées sur google, on constate que cet emploi est assez rare à l'actif; au contraire, il constitue l'essentiel des emplois attestés au passif. C'est donc bien la construction passive qui sélectionne cette valeur du verbe, c'est-àdire celle où le procès porte sémantiquement sur le second argument. En d'autres termes, peu importe la signification spécifique de monter dans tel ou tel emploi (sens de déplacement ou d'assemblage), ce qui importe pour l'emploi du passif, c'est la portée sémantique du procès sur le second argument. Cette portée se retrouve aussi bien avec la valeur de déplacement en qu'avec la valeur d'assemblage en . On constate que ces deux emplois sont pareillement passivables, par différence avec .

Ceci dit, si cette tendance est exacte, cette formulation en termes de contrainte sur la portée sémantique $\mathrm{du}$ procès est trop forte. Derrière cette contrainte particulière, se manifeste une propriété plus générale, dont elle ne constitue qu'une manifestation particulière. En effet, on trouve sur internet quelques emplois attestés au passif correspondant à l'emploi a), c'est-à-dire avec une portée du procès sur X, comme dans l'exemple suivant :

(23) Dans une de ces scènes à la chorégraphie soignée, Palma s'amuse à faire référence à la belle scène de l'escalier des Incorruptibles, elle-même inspirée d'Eisenstein. On retrouve, dans Dahlia noir, une scène à la fois même et autre, où l'escalier est monté au lieu d'être descendu, mais toujours au ralenti, et toujours avec le même suspense.

Certes, dans cet emploi, le déplacement vers le haut ne concerne pas le second argument ( l'escalier) mais une autre entité. Observons pourtant que cette entité, correspondant ici au premier argument, X, est non exprimée; c'est le cas de tous les emplois similaires que nous avons relevés. Dans cet emploi, « on analyse une scène de film et le "personnage principal » est l'escalier lui-même qui se trouve interprété comme affecté par le fait que des personnes le montent ». Le terme "affecté » est trop fort, notamment parce qu'il n'est pas transposable aux autres types de procès, comme nous le verrons ci-après.

Cependant, ce qui est juste dans l'analyse de cet emploi, c'est que le contexte induit une relation sémantique particulière entre le second argument et le procès : l'escalier est caractérisé par le fait même d'être le lieu de cet événement. La contrainte générale serait donc la suivante : le passif impose que le procès soit interprétable comme caractérisant d'une façon ou d'une autre le second argument. Dès lors, on comprend que le passif sélectionne les emplois de verbes transitionnels où la transition concerne le second argument.

Nous nous proposons maintenant de tester cette hypothèse sur les deux autres grands types de procès, à savoir les procès dynamiques non transitionnels et les procès statifs. 


\section{3 Étude des lacunes distributionnelles concernant les procès dynamiques non transitionnels}

48

Considérons les emplois suivants :

(24)a. cet été, Paul a visité la Chine

(24)b. François a suivi le match à la télé

(24)c. Marie a traversé la ville en 10 minutes "il s'est passé quelque concernant Paul / François / Marie » et non par « il est arrivé quelque chose à la Chine / à la télé / à la ville ». Dans ces trois exemples, le procès ne caractérise pas le second argument $Y$, mais le premier, $X$. On constate que l'on obtient des séquences peu naturelles au passif :

(25)a. ?? cet été, la Chine a été visitée par Paul

(25)b. ?? le match a été suivi à la télé par François

(25)c. ?? la ville a été traversée par Marie en 10 minutes

On retrouve donc la même contrainte sémantique que pour les procès transitionnels. Précisons l'analyse. Tout en gardant pour chaque verbe la signification liée aux emplois ci-dessus, quelle modification permettrait de rendre la séquence acceptable au passif ? Ces emplois deviennent parfaitement naturels au passif si l'on modifie la référence du premier argument sur le plan quantitatif et / ou notionnel :

(26)a. cet été, la Chine a été visitée par plus de 10 millions de touristes étrangers

(26)b. le match a été suivi à la télé par près de 7 millions de téléspectateurs

(26)c. chaque jour, la ville est traversée par des milliers de camions

51 Le verbe visiter est employé en avec le même sens qu'en ; par contre, concernant le premier argument, il est maintenant question de 10 millions de touristes et non plus d'un seul (changement quantitatif). Le fait que les visiteurs soient plus de 10 millions devient suffisamment saillant pour que leurs venues définissent une situation caractérisant l'état de la Chine (par exemple la Chine est un pays qui attire les touristes). Le même principe vaut pour suivre le match (un téléspectateur / 7 millions de téléspectateurs). Un match suivi par 7 millions de téléspectateurs est un match qui a eu du succès. Pour l'emploi « traverser la ville » il y a à la fois un changement notionnel (il n'est plus question d'une personne mais d'un camion) et un changement quantitatif (des milliers de camion). Ces trois exemples en révèlent un point essentiel : le premier argument lui-même, en combinaison avec le verbe, peut servir à caractériser l'état du référent du second argument.

La modification quantitative du premier argument n'est en rien obligatoire. Tout dépend des propriétés notionnelles ${ }^{12}$ de cet argument par rapport à la signification du verbe et à celle du second argument. Si X est suffisamment saillant, notamment s'il renvoie luimême à une entité dynamique qui exerce une action sur le second argument du verbe, alors l'emploi du passif redevient parfaitement naturel :

(27) la ville [Y]a été traversée par une vague de $2 \mathrm{~m}$ de haut / une tornade / une coulée de lave $[\mathrm{X}]$

Ici, les termes tornade ou coulée de lave, à travers l'événement qu'ils évoquent, permettent d'interpréter le sujet du passif comme affecté par le procès en jeu ; le référent du sujet est donc, comme dans les exemples précédents, caractérisé sémantiquement (la ville est en ruine / ravagée, etc.). Considérons encore l'exemple suivant du verbe suivre: 
(28)a. Marie suit des études de droit

(28)b. ?? des études de droit sont suivies par Marie

\subsection{Etude des lacunes distributionnelles concernant les procès statifs}

filles, en France (données 2005-2006).

(29)b. Tout le monde sait que les études de droit sont suivies en majorité par les

voyous pour apprendre la meilleure façon de contourner la loi !

Dans l'exemple a), on peut comprendre que les études de droit se féminisent (c'est en cela que le premier argument du V, en lien avec ce verbe, caractérise les études de droit). Ces deux exemples permettent de préciser notre hypothèse : la portée sémantique du procès, liée à la signification du verbe dans l'emploi considéré, n'est qu'un élément parmi d'autres qui conditionne la possibilité du passif. Ce qui demeure vrai, c'est qu'au passif le second argument du verbe doit être caractérisé par le procès. Mais l'élément caractérisant n'est pas forcément le verbe seul ; cela peut concerner le premier argument lui-même comme ci-dessus. C'est pourquoi des emplois de verbe où le procès porte sémantiquement sur le premier argument ( $X, c^{\prime}$ est-à-dire le complément d'agent) sont néanmoins acceptables au passif, à condition que $X$, en combinaison avec le verbe, soit sémantiquement à même de pouvoir caractériser l'état du référent du sujet.

Nous partirons d'un emploi du verbe habiter qui a été discuté à plusieurs reprises dans la littérature s'intéressant à l'impassivabilité de certains emplois de verbe (cf. Ruwet (1983 : 124-125 ; Gaatone (1998 : 260) ; Raineri (2010:60) :

(30)a. Philippe habite le $11^{\text {ème }}$ arrondissement.

(30)b. ?? Le $11^{\text {ème }}$ arrondissement est habité par Philippe

Observons tout d'abord que l'emploi au passif est assorti d'un effet sémantique que l'on n'a pas à l'actif: les jugements des locuteurs sur b) sont habituellement assortis de commentaires comme: "on a l'impression que Paul est seul à habiter le $11^{\mathrm{e}}$ arrondissement / qu'il occupe à lui tout seul le $11^{\mathrm{e}}$ arrondissement / etc. ». Constatons que ces effets interprétatifs sont absents de la séquence à l'actif : en lisant a), on n'en déduit pas que Paul est seul à habiter le $11^{\mathrm{e}}$ arrondissement. Au-delà de la seule différence d'acceptabilité, il y a donc un effet interprétatif qui est lié au passif luimême.

Ces intuitions des locuteurs découlent du fait qu'il est peu naturel de caractériser l'état d'un arrondissement par le fait qu'une personne y habite. Cette observation manifeste encore une fois la contrainte générale selon laquelle au passif le procès doit caractériser l'état du référent du second argument. A la suite de Ruwet, on constate que si l'on modifie le premier argument sur le plan quantitatif et notionnel, alors la séquence devient acceptable au passif : 
(31)a. un grand nombre d'étrangers habitent le $11^{\mathrm{e}}$ arrondissement

(31)b. le $11^{\mathrm{e}}$ arrondissement est habité par un grand nombre d'étrangers

Nous voyons à nouveau que les propriétés sémantiques du premier argument peuvent contribuer à caractériser l'état du référent du sujet du passif et rendre la séquence acceptable. En effet, il est plus naturel de caractériser l'état d'un arrondissement par le fait qu'un ensemble de populations y habitent.

Il convient ici de préciser un point sur les relations entre sémantique et pragmatique concernant le passif. Gaatone, à propos de ces mêmes exemples reprend une observation de Ruwet (1983) selon lequel « le passif n'est naturel que si le référent du sujet [de l'actif] « occupe » en quelques sortes « toute la place» (p. 124). Il conclut que l'impassivabilité de .b) relèverait « non pas d'un problème syntaxique et / ou sémantique, mais pragmatique " (p. 260). Raineri (2010:60) adopte une position similaire à propos de ce même exemple : «la notion de caractérisation du référent du sujet par le prédicat relève de la pragmatique plutôt que de la sémantique lexicale ».

61 Il semble qu'il y ait là une confusion dans les niveaux d'analyse. Que l'on conçoive plus facilement qu'un arrondissement soit caractérisé par le fait d'être habité par un ensemble de populations plutôt que par le fait qu'un individu y habite, cela relève en effet de considérations pragmatiques. Mais le fait même qu'au passif le référent du sujet doive être caractérisé par le procès, cela ne relève en aucun cas de la pragmatique ; cela relève d'une contrainte sémantique imposée par le passif lui-même, puisqu'encore une fois, la configuration active n'impose pas que le complément le $11^{\text {ìme }}$ arrondissement soit caractérisé par le procès. Précisons qu'ici nous parlons bien des contraintes sémantiques sur le sens de la phrase passive et non des contraintes sur l'insertion d'une phrase passive dans un texte (qui elles bien sûr relèvent de facteurs pragmatiques).

2 De plus, les relations pragmatiques entre les deux arguments du verbe habiter sont très variables dans les exemples attestés. Le référent du premier argument n'occupe pas forcément tout l'espace défini par le second argument, comme le suppose Ruwet, ainsi que le montrent les exemples attestés suivants, tirés d'internet ${ }^{13}$ :

(32)a. L'immeuble est habité par un marchand d'art de réputation internationale

(32)b. Chaque palais est habité par un esprit supérieur

Ici, les propriétés notionnelles du premier argument sont suffisamment saillantes pour que sa seule présence suffise à caractériser le lieu, même s'il n'occupe pas le lieu en entier. Peu importe que $\mathrm{X}$ occupe ou pas tout l'espace défini par $\mathrm{Y}$, il faut simplement qu'au passif le sujet $(\mathrm{Y})$ soit caractérisé par le participe passé et / ou / et le premier argument du verbe.

4 On peut multiplier les observations. Elles aboutissent invariablement à la même conclusion : pour rendre la séquence acceptable au passif, il faut que les déterminations sémantiques $\mathrm{du}$ verbe et éventuellement celles de son premier argument puissent s'interpréter comme caractérisant l'état du référent du sujet. Partons des séquences suivantes, où le procès caractérise le premier argument :

(33)a. Marie danse très bien le tango

(33)b. Cécile parle couramment l'anglais

(34)a. ?? le tango est très bien dansé par Marie

(34)b. ?? l'anglais est parlé couramment par Cécile

65 Il suffit de modifier sémantiquement le premier argument afin qu'il puisse s'interpréter comme caractérisant l'état du référent du second argument, pour que ces emplois de verbes deviennent acceptables au passif : 
(35)a. En Europe, le tango est dansé par des gens aisés.

(35)b. l'anglais est parlé par plus de 600 millions de personnes à travers le monde

\subsection{Observations supplémentaires}

Reprenons les deux exemples vus ci-avant :

(33)a. Marie danse très bien le tango

(33)b. Cécile parle couramment l'anglais

La modification sémantique du premier argument n'est pas la seule possibilité pour rendre la séquence acceptable au passif. On peut laisser indéterminé le premier argument du verbe et ajouter un complément circonstanciel. Dès lors que le contenu sémantique de ce complément permet de caractériser le référent du sujet du passif, on obtient à nouveau une séquence acceptable au passif, comme dans les exemples attestés suivants :

(36)a. L'anglais est parlé dans le monde entier

(36)a'. Dans la ville, l'anglais est parlé avec un accent très particulier

(36)b. D'ailleurs, le tango est dansé de façon très rapproché, il est provocateur, explicite.

68 Dans la première séquence, le complément circonstanciel de lieu, en lien avec le verbe, caractérise l'état du référent du sujet, dans la mesure où il signifie dans ce contexte que l'anglais est une langue très répandue; dans la deuxième séquence le complément de manière explicite la façon dont l'anglais est parlé, ce qui caractérise à nouveau le référent du second argument. Enfin, dans la dernière séquence, là aussi le complément de manière, en lien avec le verbe danser, caractérise le référent du second argument le tango (le tango est une danse sensuelle, etc.).

69 Nous allons maintenant aborder des emplois où le second argument renvoie lui-même à un procès dynamique, cas de figure peu étudié avec le passif, à notre connaissance. Le rôle caractérisant des compléments circonstanciels y apparaît de façon encore plus nette. Considérons l'emploi suivant du verbe ressentir :

(37)a. Marie a ressenti la secousse sismique

(38)b. ? la secousse sismique a été ressentie par Marie

70 Cet emploi de ressentir évoque un fait perceptif à propos d'un événement exprimé par le second argument (secousse sismique). Le procès ressentir caractérise plutôt le premier argument ; on comprend qu'il s'est passé quelque chose concernant Marie et non qu'il s'est passé quelque chose concernant la secousse sismique. On retrouve à nouveau la même contrainte sur l'emploi du passif. Comme dans les exemples précédents, on peut rendre la séquence plus naturelle au passif en modifiant le premier argument sur le plan quantitatif :

(38) la secousse a été ressentie par tous les habitants de la région

71 Cette modification quantitative permet de caractériser la secousse, par exemple comme étant une secousse de forte puissance, satisfaisant ainsi à la contrainte imposée par le passif.

72 Remarquons au passage les limites de la terminologie traditionnelle concernant les termes de passif et complément d'agent : ici, c'est le complément dit d'agent qui subit l'événement exprimé par le sujet du passif !

Revenons à l'exemple. La séquence devient également acceptable au passif si on ajoute des éléments qui ne font pas partie de la structure argumentale du verbe, comme dans les exemples attestés suivants : 
(39)a. La secousse a été ressentie jusqu'à Tokyo, à $163 \mathrm{~km}$ plus au sud

(39)b. la secousse a été ressentie pendant environ cinq minutes, en pleine après-

midi

(39)c. la secousse a été ressentie dans 15 communes durant quelques secondes

74 Dans tous ces emplois, le premier argument du verbe n'est pas exprimé. Les différents éléments ajoutés correspondent à des compléments circonstanciels, soit à valeur spatiale, pour le premier exemple, soit à valeur temporelle pour le suivant, soit une combinaison des deux types, pour le dernier. A chaque fois, il s'agit de caractériser la secousse, soit dans son étendue, soit dans sa durée et / ou son intensité. Certains emplois expriment la prédication d'existence elle-même de la secousse :

(40) Une forte secousse a été ressentie lundi à New Delhi

Le sens de cet emploi est proche de il y a eu une forte secousse lundi à New Delhi.

\subsection{Bilan}

76 A l'issue de ce parcours d'observation, allant des procès transitionnels au procès statifs, nous constatons que dans tous les cas, le passif impose que le référent du sujet (le second argument $(\mathrm{Y})$ ) soit caractérisé sémantiquement par le participe passé combiné éventuellement à d'autres éléments de la proposition. Dans tous les cas, lorsqu'un emploi ne satisfait pas cette contrainte, il est difficilement passivable.

Nous avons constaté que les éléments qui caractérisent le référent du second argument au passif sont extrêmement variables et peuvent également correspondre à des compléments circonstanciels. Nous avons donc trois grands cas de figure, selon que le second argument est caractérisé par :

- le verbe seul : la course a été annulée- le verbe et le premier argument :

la course a été regardée par des millions de téléspectateurs enthousiastes

- le verbe et un ou des complément(s) circonstanciel(s) :

la course a été organisée pour la première fois en Inde en 2004.

En résumé, peu importent les autres compléments phrastiques qui sont mobilisés pour caractériser le sujet du passif. Ce qui compte, c'est que le sujet soit caractérisé par ces compléments. Il apparaît donc que l'acceptabilité d'une séquence au passif dépend de multiples facteurs, selon les propriétés sémantiques du verbe, celles de ses compléments, la relation sémantique entre le verbe et le sujet syntaxique, et la présence éventuelle de compléments circonstanciels. Mais ces multiples facteurs se ramènent toujours à la même contrainte sémantique générale : l'état du référent du sujet du passif doit pouvoir être interprété comme caractérisé par le verbe et ses éventuels modifieurs. En conclusion, l'ensemble des lacunes distributionnelles examiné ci-avant manifeste une régularité sémantique.

De plus, cette régularité sémantique révèle une dissymétrie entre l'actif et le passif. A l'actif, selon les emplois de verbe, on peut comprendre que le second argument est caractérisé ou pas. Au passif, le verbe et ses éventuels modifieurs doivent toujours caractériser le second argument. Cette régularité sémantique permet donc de donner une assise empirique à l'intuition classique sur le passif selon laquelle son emploi est plus contraint que l'actif : 


\begin{tabular}{|l|l|l|l|}
\hline \multicolumn{2}{|l|}{} & $\begin{array}{l}\text { le verbe et ses éventuels modifieurs } \\
\text { ne caractérisent pas le second } \\
\text { argument Y }\end{array}$ & $\begin{array}{l}\text { le verbe et ses éventuels } \\
\text { modifieurs caractérisent le } \\
\text { second argument Y }\end{array}$ \\
\hline \multirow{2}{*}{ ACTIF } & $\begin{array}{l}\text { procès } \\
\text { dynamiques }\end{array}$ & $\begin{array}{l}\text { monter les escaliers en courant } \\
\text { arrêter le droit }\end{array}$ & $\begin{array}{l}\text { hisser le drapeau } \\
\text { arrêter le fugitif }\end{array}$ \\
\cline { 2 - 5 } & procès statifs & $\begin{array}{l}\text { craindre l'humidité } \\
\text { mesurer 1m80 }\end{array}$ & $\begin{array}{l}\text { entourer la propriété } \\
\text { PASSIF } \\
\text { procès }\end{array}$ \\
\cline { 2 - 3 } & $/$ & $\begin{array}{l}\text { le drapeau a été hissé } \\
\text { le fugitif a été arrêté }\end{array}$ \\
\hline procès statifs & $/$ & $\begin{array}{l}\text { la propriété est entourée par une } \\
\text { clôture électrifiée }\end{array}$ \\
\hline
\end{tabular}

Précisons que la différence mise au jour ici ne relève pas du niveau de la structure informationnelle. En effet, sur ce plan, comme l'a montré Gaatone (1998), on observe les mêmes variations qu'à l'actif: le sujet du passif peut correspondre à un élément présupposé ou pas, connu ou pas, en topique ou pas (au sens de Lambrecht 1994), etc. Voici deux exemples de passif avec sujet non topique, correspondant à ce que Lambrecht appelle des prédications « event-reporting » (Lambrecht, id., p. 124) où l'ensemble de la relation prédicative fait partie du « focus $»^{14}$.

(41)a. 25 févr. 2011 ... Joggeuse disparue: un homme a été placé en garde à vue.

(41)b. 24 août 2010 ... Aucun des habitants n'étaient présents sur le lieu, seul un

chien a été découvert inconscient et asphyxié par l'épaisse fumée noir

Reprenons maintenant l'analyse du passif. Nous allons centrer notre analyse sur le statut de l'élément être qui, selon les études, est considéré comme un simple auxiliaire ou bien comme un verbe lexicalement plein. Nous allons voir que la régularité sémantique mise au jour dans cette première partie conforte le statut de verbe plein de être au passif. Mais, dans un premier temps, nous allons préciser les termes du débat.

\section{Les données aspectuelles, syntaxiques et distributionnelles confortent l'analyse lexicale du passif}

\subsection{Introduction}

Nous considérerons que le passif, au sens défini ici, est un objet empirique porteur en soi d'une signification, au même titre qu'un morphème ou un mot. Sur ce point, notre approche rejoint celle des grammaires de construction : " constructions-form-meaning correspondences that exist independently of particular verbs. That is, it is argued that constructions themselves carry meaning, independently of the words in the sentence." (A. Goldberg, $1995: 1)^{15}$. Mais, ce positionnement n'est pas spécifique aux grammaires de construction, on le retrouve par exemple dans la théorie des opérations prédicatives et énonciatives initiée par A. Culioli (cf. Culioli, [1982] 1999). 
Si l'on admet que le passif a un sens, se pose alors la question de la compositionnalité éventuelle de ce sens par rapport aux éléments constitutifs de cette construction. Cette question concerne avant tout le statut de l'élément être. La tradition grammaticale, généralement reprise par les linguistes, considère l'élément être au passif comme un simple auxiliaire, une marque formelle du passif, sans signification en propre (cf. Gaatone, 1998 : 12-14 ; Helland, $2002: 67$; Carlier 2002). Cela sous-entend que le sens du passif n'est pas compositionnel par rapport à la présence de l'élément être.

C'est la position défendue par l'analyse sémantique du passif proposée dans Raineri (2010), dans le cadre du modèle de grammaire de construction de A. Goldberg (1995): "[il] va de soi que le sens passif de [...]ETRE Vé, s'il existe, ne peut être dit compositionnel, c'est-à-dire qu'il ne peut être considéré comme la somme de "be/être " et du participe passé Ven/Vé, puisqu'il serait alors identique au sens de la construction attributive homonyme»(p.347). Certes, Raineri nuance aussitôt son propos (id.) : « Toutefois, [...], les constructions attributives sont sémantiquement liées au passif en [...] ETRE Vé, ce qui signifie que la nature même de ces deux composants joue bien évidemment un rôle décisif dans le sens passif de ces formes ». Néanmoins, l'auteur ne propose pas d'analyse sémantique compositionnelle du passif par rapport à ses éléments constitutifs.

5 Quelques auteurs attribuent à l'élément être du passif un statut intermédiaire entre auxiliaire et verbe plein ; par exemple, Kronning (2003) le considère comme un coverbe non auxiliaire ${ }^{16}$. Enfin, quelques auteurs lui attribuent un statut de verbe plein (cf. Milner : 1985 ; Abeillé et Godard : 1996, 2002 ; Herslund et al. : 1997), sous-entendant que le sens du passif est compositionnel par rapport à la présence de l'élément être.

6 Ce débat pourrait être reformulé en termes de degré de grammaticalisation, en particulier dans le cadre du parcours de grammaticalisation «Verb-to-TAM ${ }^{17}$ » de B. Heine (1993). Si être est un simple auxiliaire du passif, cela revient à le considérer comme fortement grammaticalisé ; si au contraire, au passif, être est un verbe plein, cela revient à considérer qu'il est peu ou pas grammaticalisé. A notre connaissance, les auteurs s'étant intéressés à être au passif ne le décrivent généralement pas en ces termes, hormis Buchard (2009). Celle-ci considère qu'au passif la combinaison être + participe passé connaît un «haut degré de grammaticalisation» (p. 276). En tout état de cause, la notion de grammaticalisation donne lieu à de nombreux débats que nous n'aborderons pas ici.

Comme nous l'avons indiqué en introduction, notre préoccupation dans cet article est avant tout d'ordre descriptif. Nous cherchons à établir dans quelle mesure les propriétés de l'élément être au passif correspondent à celles du verbe être lexicalement plein, c'est-àdire non grammaticalisé. Comme nous l'avons rappelé en introduction, le passif en français a été étudié de multiples points de vue. Le problème, selon nous, est que ces différents points de vue ne sont généralement pas articulés. Par exemple, on trouve des études sur les propriétés aspectuelles du passif (Carlier, 2002) mais qui ne prennent pas en compte ses propriétés syntaxiques et sémantiques. On relève des études syntaxiques du passif, mais qui ne prennent pas en compte, ou très marginalement, ses dimensions aspectuelle et sémantique (Abeillé et Godard, 1996, 2002). On relève par ailleurs des études sémantiques du passif mais qui ne prennent pas en compte ses propriétés syntaxiques et aspectuelles (Raineri, 2010). Lorsque ces différents angles d'observation sont abordés dans une même étude, ils le sont de façon également isolée (cf. Gaatone, 1998). 
découpage du passif en plans d'observation isolés les uns des autres empêche selon nous de voir une cohérence dans les différentes propriétés de être au passif. Nous défendons ainsi la nécessité de combiner des observations relevant des trois dimensions suivantes du passif :

- des observations concernant les propriétés aspectuo-temporelles du passif

- des observations concernant les propriétés syntaxiques du passif

- des observations concernant les propriétés sémantiques du passif, en l'occurrence la propriété sémantique mise au jour dans l'étude des lacunes distributionnelles, dans la première partie.

89 Nous allons voir que leur articulation permet de mettre en évidence une convergence de faits à l'appui de l'analyse lexicale de l'élément être au passif (i.e. en faveur de son statut peu ou pas grammaticalisé).

\subsection{On retrouve au passif les propriétés aspectuo-temporelles du verbe être}

A la suite de Abeillé et Godard (1996) et (2002), rappelons tout d'abord que, sur le plan temporel, la séquence être + Vpp au passif a la même valeur que le verbe être et se distingue d'emblée de la valeur temporelle du passé composé :

(42)a. Jean est allé à Paris

(42)b. Jean est attendu à Paris

(42)c. Jean est restaurateur à Paris

91 Alors que la séquence a) correspond à une valeur temporelle révolue, les séquences b,c) qui correspondent respectivement au passif et au verbe être avec attribut renvoient toutes les deux au présent. La valeur temporelle de être au passif est donc identique à sa valeur en tant que verbe plein.

Examinons maintenant les faits sur le plan aspectuel (grammatical et lexical). A la suite de Carlier (2002), partons du contraste aspectuel bien connu entre actif et passif lorsqu'on utilise le présent avec un procès dynamique télique :

(43)a. on sert le vin

(43)b. le vin est servi

93 A l'actif, le procès a une représentation aspectuelle inaccomplie (on présente l'action en train de se dérouler), alors qu'au passif, hors cotexte supplémentaire, l'interprétation la plus naturelle donne le procès comme accompli (ça y est, c'est fait). L'explication traditionnelle la plus courante repose sur l'hypothèse que l'on a une valeur d'accompli car la forme de participe passé marquerait elle-même l'aspect accompli. Carlier conteste cette hypothèse en s'appuyant notamment sur le fait que lorsque le procès est de type statif, cette valeur d'accompli ne s'observe pas :

(44)a. Pierre aime Marie

(44)b. Marie est aimée de Pierre

La séquence b) n'a en aucun cas une interprétation accomplie. On comprend que Pierre aime toujours Marie. On aurait par contre une valeur d'accomplie avec le passé composé dans Marie a été aimée de Pierre (au sens où le déroulement du procès est présenté comme terminé18), mais ni plus ni moins qu'à l'actif dans Pierre a aimée Marie. L'émergence de la valeur d'accompli en b) ne peut donc provenir du participe passé lui-même ${ }^{19}$. 
Carlier fonde son explication à partir de l'hypothèse qu'au passif, on retrouve la valeur aspectuelle de être qui, en tant que verbe, a une dimension fondamentalement stative. Dans le cadre du modèle de la temporalité développé par Gosselin (1996), Carlier considère qu'en b) nous avons un conflit interprétatif entre d'une part l'aspect lexical associé à cet emploi du verbe (exprimant un nouvel état à atteindre et donc un changement) et d'autre part l'aspect statif (pas de changement) construit par le verbe être . La résolution du conflit interprétatif passe par la construction de la représentation de la phase résultative du procès ${ }^{20}$.

La différence aspectuelle entre l'actif et le passif pour les procès dynamiques téliques découle de cette valeur stative du verbe être et non d'une propriété du participe dit « passé ».

Cette analyse permet également de rendre compte du fait que pour les procès de type statif, il n'y a pas de changement aspectuel entre actif et passif (cf. . Ici il n'y a pas de conflit interprétatif : l'aspect lexical du verbe de l'actif est identique à l'aspect lexical du verbe être.

Buchard (2009), reprenant et étendant les observations de Carlier (2002) aboutit à la même conclusion (p. 64) : quel que soit le type de procès associé au Vpp, le passif tend systématiquement à privilégier une interprétation stative, c'est-à-dire la valeur aspectuelle de être en tant que verbe plein. Cette tendance est difficile à expliquer si on considère que l'élément être au passif est un simple auxiliaire sans rapport avec les propriétés lexicales du verbe être.

\subsection{On retrouve au passif les propriétés syntaxiques du verbe être}

Par définition, si une forme verbale correspond à un auxiliaire, alors le verbe qui la suit ne peut pas constituer un complément de cette forme verbale (dans il a mangé, l'élément verbal mangé ne correspond pas à un complément de l'auxiliaire a). Donc, sur le plan synytaxique, si l'on considère que la forme être au passif est un simple auxiliaire, le participe passé ne peut pas correspondre à complément de être.

Un argument confirme à première vue le statut d'auxiliaire de l'élément être au passif : la monté des clitiques. Lorsqu'un verbe se combine avec un auxiliaire et que l'on pronominalise par un clitique le complément de ce verbe, alors le pronom clitique ne se place pas devant le verbe mais «monte» devant l'auxiliaire, comme dans l'exemple suivant:

(45)a. ce matin, Jean a parlé à la directrice

(45)b. * ce matin, Jean a lui parlé

(45)c. ce matin, Jean lui a parlé

01 A l'inverse, lorsque le premier verbe n'a pas le statut d'auxiliaire, alors le pronom clitique doit se placer directement devant le deuxième verbe, comme dans l'exemple suivant :

(46)a. ce matin, Jean veut parler à la directrice

(46)b. ce matin, Jean veut lui parler

(46)c. * ce matin, Jean lui veut parler

Or, au passif, le pronom clitique complément du participe passé ne se place pas devant ce dernier mais doit «monter» devant être, laissant supposer que celui-ci se comporte comme un auxiliaire : 
(47)a. ce cadeau est offert à Marie par toute l'équipe

(47)b. * ce cadeau est lui offert par toute l'équipe

(47)c. ce cadeau lui est offert par toute l'équipe participe passé « a un caractère adjectival » (p. 43) et a donc une interprétation stative. Or, A. Buchard (2009: 236-238), montre, sur un corpus de plus de 200 verbes, qu'il n'en est rien. Il y a tout autant ou davantage de séquences acceptables avec des participes passés correspondant à un procès dynamique, donc clairement verbal, qu'avec des participes passés correspondant à un procès statif. En l'occurrence, dans l'exemple b) ci-dessus le participe passé (applaudies) correspond à un procès dynamique. Voici quatre autres exemples avec un procès dynamique parfaitement acceptables fournis par Buchard (2009) :

(51)a. Ces documents peuvent être utilisés comme l'est généralement le manuel dans une classe de langue.

(51)b. Charlot qui s'est frayé un passage en franchissant des voies interdites est sermonné comme le serait un enfant

(51)c. Car une constatation peut être discutée, et doit l'être [...]. (Alain, Propos)

(51)d. Le pétrole qui est acheté, l'est par le biais d'une résolution "pétrole contre nourriture". 
110 Ces données montrent que le participe passé est un complément de être. Ce dernier ne peut donc pas être considéré comme un auxiliaire. Il correspond donc nécessairement à un coverbe non auxiliaire ou bien à un verbe plein. Abeillé et Godard (2002) font l'hypothèse qu'il correspond à la copule et que le participe passé (et ses éventuels modifieurs) correspond à un attribut du sujet. La particularité de cet attribut est qu'il est constitué par un syntagme verbal (puisque le participe passé est une forme du verbe).

L'hypothèse d'un attribut du sujet de nature verbal fait débat puisque, par définition, l'attribut correspond à un complément non verbal. On peut alors soi décider d'élargir la notion d'attribut soi considérer que le participe passé correspond à un autre type de complément du verbe être.

112 Ce débat excède les limites de cet article. Notons brièvement que l'hypothèse du statut d'attribut est au moins partiellement défendable en raison du statut catégoriellement hybride du participe passé au passif: il a un fonctionnement partiellement verbal et partiellement adjectival (Abeillé et Godard n'argumentent pas sur ce point). En effet, comme l'a montré N. Rivière (1990) le participe passé au passif peut se combiner avec l'adverbe très; or, cet adverbe ne se combine pas avec des verbes mais avec des adjectifs, alors que l'adverbe beaucoup se combine au contraire avec des verbes :

(52)a. ces événements ont beaucoup / *très affecté Marie

(52)b. Marie a été ? beaucoup / très affectée par ces événements

(52)c. Marie est * beaucoup / très émue

113 Ceci dit, en tant que verbe, être admet au moins deux types de complément: le complément attribut (Paul est sympathique / Paul est médecin) et le complément locatif (Paul est à Paris $)^{22}$. Dans le principe, rien n'empêche d'envisager pour ce verbe un troisième type de compléments, de nature verbale. Quoi qu'il en soi, nous nous contenterons ici de parler du participe passé comme simple complément du verbe être, sans préciser les relations éventuelles avec les autres types de compléments du verbe être.

\subsection{On retrouve au passif les propriétés sémantiques du verbe être}

Nous avons maintenant rassemblé suffisamment d'observations pour aborder la discussion sur l'analyse sémantique du passif. Quel que soit le cadre théorique adopté, et quelle que soit l'analyse sémantique que l'on propose pour le passif, celle-ci doit être compatible d'une part avec les propriétés aspectuelles et syntaxiques du passif, d'autre part avec la régularité sémantique manifestée par les lacunes distributionnelles.

\subsubsection{La régularité sémantique manifestée par les lacunes distributionnelles correspond au sens du verbe être}

115 Rappelons la régularité sémantique mise au jour dans la partie 2 à propos des lacunes distributionnelles : dans tous les cas, le passif impose que le référent du sujet (le second argument $(\mathrm{Y})$ ) soit caractérisé sémantiquement par le participe passé combiné éventuellement à d'autres éléments de la proposition.

Cette hypothèse sémantique correspond directement à la description sémantique généralement associée à la fonction attribut avec le verbe être. Ainsi, Riegel (1984:31) parle de la «prédication d'une caractéristique; plus loin: «la propriété [exprimée par l'attribut] caractérise [le sujet] » (p. 38). A. Buchard (2006: 69), décrit l'attribut de l'objet comme « une seconde prédication caractérisant l'état du référent du syntagme nominal 
objet concomitant à l'action verbale ». De même, P. Lauwers et E. Tobback (2010: 80) considèrent que ce complément « attribue une caractéristique à un référent ». P. Lauwers et L. Melis (2013: 252) parlent de "prédication caractérisante".

Plus précisément, ces deux derniers auteurs distinguent deux grandes valeurs sémantiques de l'attribut: une valeur caractérisante que l'on rencontre principalement avec les adjectifs qualificatifs, et une valeur catégorisante que l'on rencontre avec les noms. Le fonctionnement sémantique du passif serait donc proche de celui des attributs adjectivaux. Cela est cohérent avec la dimension adjectivale du participe passé que nous avons rappelé en 3.4.

Ainsi, l'analyse sémantique des lacunes distributionnelles confirme le statut de verbe plein de l'élément être au passif. Cette analyse sémantique revient à considérer que la signification du passif se définit fondamentalement dans la relation entre le sujet (second argument du verbe) et le verbe, plutôt qu'entre le verbe et son complément d'agent (nous y reviendrons en 3.5.). Sur ce point, notre analyse rejoint celle proposée par A. Culioli ([1971] 1999 ; ([1982] 1999). Elle s'en distingue dans le fait de ne pas définir le passif par rapport à l'actif qui en serait d'une façon ou d'une autre le point de départ. En effet, dans Culioli ([1971] 1999) et Culioli ([1982] 1999) le passif est défini comme un changement d'orientation de la relation prédicative à partir d'une orientation primitive: «Si l'on inverse la relation afin de prendre comme départ ce qui était l'arrivée, il se produit une dissymétrie: sur le nouveau terme de départ, on prédique une propriété, puis, éventuellement, on adjoint l'agent qui a provoqué ce nouvel état » ([1971] 1999: 40 ${ }^{23}$.

Elle s'en distingue aussi dans la description sémantique du phénomène. En effet, comme nous l'avons vu ci-avant, le passif ne correspond pas toujours à la prédication d'une propriété ni à un changement d'état (notamment avec les procès statifs). La notion de " caractérisation du référent du sujet » intègre ces deux aspects (propriété / changement d'état) tout en étant sémantiquement plus large.

\subsubsection{Limites d'une analyse sémantique basée sur la transitivité prototypique} (Raineri, 2010)

L'analyse sémantique proposée par Raineri (2010) est la seule, à notre connaissance, à articuler explicitement l'observation des lacunes distributionnelles et l'analyse sémantique du passif. Elle aborde le passif comme une construction symétrique de l'actif. Ceci a pour conséquence que les emplois jugés prototypiques dans la transitivité sémantique sont également ceux qui sont jugés prototypiques au passif.

Selon l'auteur (2010: 355), le passif met prototypiquement en jeu un procès dynamique impliquant un agent et un patient. Cela a pour conséquence qu'au passif le sujet doit être affecté par le procès. Cette hypothèse sur le passif en français est la reprise d'une analyse sémantique du passif en anglais proposée par Bolinger (1975:67): "the subject in a passive construction is conceived to be a true patient, i.e., to be genuinely affected by the action of the verb. If the grammatical object in the active construction is not conceived as a true patient, there will be no corresponding passive".

Cette analyse présente une double difficulté, d'une part concernant la description des lacunes distributionnelles, d'autre part concernant l'analyse du passif lui-même.

Concernant la description des lacunes distributionnelles, cette hypothèse prototypique est selon nous trop forte. Elle amène Raineri à des aménagements selon nous artificiels dans le cas des procès statifs où précisément il n'y a pas de patient : " tous les énoncés 
passifs qui viennent d'être étudiés sont possibles parce que l'extensibilité de la notion d'affect attachée au rôle patient de la construction passive permet de faire fusionner celui-ci avec un participant qui n'est pas à strictement parler un patient mais est métaphoriquement conçu comme tel» (p.362). Plus loin l'auteur précise: «la construction passive a pour effet de " patientiser » l'entité-cible du procès en vertu de sa structure sémantique. Cette " patientisation » peut prendre un caractère très abstrait, car la notion d'affect sur laquelle repose le sens de la construction est particulièrement extensible». (p. 368). L'auteur en vient à vider la notion de patient de sa substance, ce qui lui enlève toute portée explicative. En particulier, cette analyse ne permet pas de rendre compte de la non corrélation, vue en section 2.1., entre degré de passivabilité et degré de transitivité (puisque le passif prototypique correspond au prototype de la transitivité sémantique).

Dans notre analyse des lacunes distributionnelles, l'affectation du second argument par le procès dans certains emplois n'est qu'une conséquence sémantique locale dans le cas où nous avons affaire à un procès dynamique télique mettant en jeu un changement (la table a été renversée / déplacée / rabotée / etc.). En effet, puisqu'au passif le procès exprimé par le verbe (en combinaison avec ses éventuels modifieurs) doit caractériser $\mathrm{Y}$, alors, quand le procès met en jeu un changement de situation, ce changement de situation doit concerner $Y$; d'où l'émergence de la contrainte sémantique locale "Y est affecté par le procès ». Mais dans le cas des procès statifs, il n'y a pas de «patientisation» du sujet; il y a simplement caractérisation du sujet du passif par le procès statif.

Concernant l'analyse du passif, l'hypothèse de Raineri revient à considérer que la dynamicité du procès est au cœur du sémantisme de cette construction (puisque ce serait sa valeur prototypique). Or, nous avons vu en 3.2. que le passif, par différence avec l'actif, privilégie au contraire l'interprétation stative des procès dynamiques téliques. L'analyse de Raineri ne permet pas d'en rendre compte. Si l'on admet au contraire que le sémantisme du verbe être est au cœur de la signification du passif, les contraintes aspectuelles observées ci-avant s'expliquent naturellement: l'affinité du passif avec l'aspect lexical statif découle de la valeur stative du verbe être.

\subsection{Retour sur le statut du complément d'agent}

126 A l'issu de ce parcours, nous pouvons reprendre le débat concernant le statut du complément d'agent au passif. Comme rappelé dans l'introduction, on peut distinguer deux grandes tendances opposées, à propos du complément d'agent: les approches considérant que le passif correspond à la perte d'un argument pour le verbe, en l'occurrence perte du premier argument (nous aurions donc une réduction de la valence du verbe); les approches considérant que la valence du verbe ne change pas au passif mais que son premier argument est mis à l'écart de la relation prédicative, marginalisé, d'une façon ou d'une autre. Il ne disparaît pas mais change de statut syntaxicosémantique ${ }^{24}$.

Sans entrer dans le détail ici, reprécisons brièvement les termes du débat. Les analyses en faveur de l'hypothèse de la réduction valencielle s'appuient notamment sur le fait que la majorité des passifs attestés sont sans complément d'agent exprimé, et que la plupart du temps, lorsque celui-ci est présent, il est facultatif, ce qui le rapproche des compléments circonstanciels. 
Cette approche rencontre plusieurs problèmes. Sans entrer dans une discussion détaillée ici, notons, ainsi que le rappelle Gaatone (1998: 186), que le complément d'agent « est sous-catégorisé par [1]e lexème verbal exactement comme l'est le sujet du verbe à la forme active ", ce qui appuie l'hypothèse que le complément d'agent correspond à un argument du verbe.

D'autre part, C. Muller (2000) souligne que les analyses défendant l'hypothèse de la réduction valencielle, essentiellement fondées sur des considérations syntaxiques dissociées de l'analyse du sens de l'énoncé, ne prennent pas en compte le type de différence sémantique illustrée par les deux séquences suivantes :

(53)a. la branche a cassé

(53)b. la branche a été cassée

Peu importe qu'en b) aucun agent ne soit exprimé. Le contraste interprétatif entre a) et b) fait apparaître que dans la deuxième séquence l'interprétation met nécessairement en jeu l'intervention d'un participant, quel qu'il soit (le vent, les enfants, un véhicule, etc.). Donc, sémantiquement, il y a bien deux arguments en jeu, l'un d'eux n'étant pas exprimé. Nous souscrivons à cette analyse, qui concerne la valence du verbe.

Mais cette hypothèse du maintien de la valence du verbe au passif est associée à une autre hypothèse, d'ordre sémantique. Selon cet auteur le passif est un " procédé grammaticalisé de mise à l'écart de l'agent»Muller (2000: 49); plus précisément (p. 51), le passif correspond à un «changement (changement par rapport à l'ordre canonique dans le verbe simple) de " point de vue » de l'action verbale, changement qui relègue au rang des relations facultativement réalisées la relation prédicative entre le premier argument et le verbe ».

2 La formulation de Muller ainsi que les termes mêmes du débat sur le statut du complément d'agent reposent, nous semble-t-il, sur un amalgame des niveaux d'analyse syntaxique et sémantique, ce qui rend ce débat en partie artificiel. En effet, lorsque l'on dit que de l'actif au passif, le premier argument passe du statut obligatoire à facultatif, cela correspond à un changement syntaxique. Ce changement découle du fait que cet argument passe d'une position syntaxique obligatoirement remplie (en français, le sujet d'un verbe conjugué est obligatoirement exprimé) à une position syntaxique facultativement remplie. Ici, l'opposition obligatoire / facultatif ne concerne pas un changement de propriétés de l'argument lui-même dans sa relation au verbe, mais correspond à une différence de propriété des positions syntaxiques occupées par cet argument.

3 Sur le plan sémantique, entre l'actif et le passif, le premier argument n'est ni plus ni moins obligatoire par rapport au sens du verbe. Considérons les séquences le lion a blesséle chasseur / le chasseur a été blessé par le lion. Dans les deux séquences, le premier argument le lion a le même rôle sémantique agentif et il est ni plus ni moins présent dans la représentation sémantique du procès.

Plus précisément, il ne faut pas confondre ce que le passif impose et ce que le passif permet. Or, dans les emplois suivants, le complément d'agent n'est pas mis à l'écart, il est au contraire mis au premier plan :

(54)a. Marie a été bousculée par le fils des voisins, complètement ivre.

(54)b. Paul a été secoué par la terrible nouvelle.

(54)c. Le voleur était entouré par une foule en colère. 
Ainsi, la mise à l'écart du complément d'agent est toute relative selon les exemples. Le passif permet une mise à l'écart du complément d'agent, mais il ne l'impose pas ; sinon, on devrait l'observer dans tous les emplois. En conséquence, la propriété de mise à l'écart de l'agent n'a pas le même statut que la propriété de caractérisation du référent du sujet mise en évidence en 2. La première est facultative alors que la seconde est obligatoire ${ }^{25}$.

Dès lors, le passif favorise les interprétations où le procès ne porte pas sur le premier argument mais sur le second; c'est ainsi que dans les emplois sur internet, pour les escaliers ont été montés, la valeur "assembler» est systématiquement privilégiée par rapport à la valeur « déplacement vers le haut. C'est le fait que la portée sémantique du procès soit (dé)tournée vers le sujet (le second argument), conséquence de la présence du verbe être au passif, qui permet secondairement une mise à l'écart du premier argument par rapport au procès.

\section{Bilan}

137 Dans un premier temps, nous avons repris l'analyse des lacunes distributionnelles au passif en français, généralement considérées comme des idiosyncrasies lexicales. Nous avons montré que ces lacunes distributionnelles étaient régulières, et que cette régularité était de nature sémantique. Selon cette propriété sémantique, le participe passé (et d'éventuels modifieurs et compléments circonstanciels de la proposition) caractérisent le référent du sujet, c'est-à-dire le second argument du verbe au participe passé. Nous avons mis au jour cette régularité sémantique aussi bien pour les procès statifs que dynamiques.

Dans un deuxième temps, nous avons repris l'analyse du passif proprement dit. Dans cette analyse, nous avons avant tout voulu montrer une cohérence entre des ensembles de faits généralement abordés de façon isolée les uns des autres, et ceci autant que possible en deçà de tout parti pris théorique ; non pas par refus de toute théorisation, mais parce que bien des prises de position dans les différents débats sur le passif se retrouvent dans des cadres théoriques très différents. Mais c'est surtout parce que l'analyse proposée ici correspond à un travail en cours d'élaboration et que nous n'avons pas encore une vision théorique d'ensemble sur cet objet complexe. Quoi qu'il en soi, nous avons voulu montrer une convergence entre des données relevant de la sémantique lexicale (à travers l'étude des lacunes distributionnelles), de la sémantique grammaticale (étude de l'aspect), et de la syntaxe. Les données de ces trois domaines convergent vers l'hypothèse que l'élément être au passif n'est pas une simple marque formelle du passif, vide de toute propriété, mais qu'il correspond au verbe être, tel qu'on peut le rencontrer dans la construction attributive. De ce point de vue, nous défendons la nécessité d'intégrer ces différents plans d'analyse dans l'étude du passif.

Alors que le passif est généralement traité comme une construction symétrique de l'actif, nous avons insisté sur la dissymétrie entre les deux constructions, du point de vue de leurs propriétés distributionnelles et sémantiques. Cette analyse permet de rendre compte de la non corrélation, évoquée en introduction, entre degré de passivabilité et degré de transitivité d'un emploi de verbe. Alors que les hypothèses sur la transitivité sémantique considèrent généralement comme prototypique les emplois correspondant à des procès dynamiques agentifs et corrélativement que les emplois passifs prototypiques mettent également en jeu cette transitivité prototypiques, notre analyse montre au contraire que c'est la stativité manifestée par être qui est au cœur du sémantisme du 
passif, les emplois exprimant un procès dynamique agentif étant finalement atypiques de ce point de vue.

Il reste néanmoins une dimension que nous n'avons pas pu aborder dans cette étude, à savoir les contraintes qui découlent de la position de sujet syntaxique elle-même. En effet, un argument du verbe dans cette position subit des contraintes sur ses propriétés référentielles (cf. Keenan 1976). Il semble ainsi que lorsque le second argument du verbe n'est pas référentiellement autonome par rapport au premier argument, l'emploi du passif est peu acceptable, même si le procès caractérise le second argument : l'inspecteur allongea le pas /?? le pas fut allongé par l'inspecteur (les pas sont les pas de l'inspecteur, ils n'ont pas d'existence autonome). Cette contrainte devra faire l'objet d'une analyse détaillée pour établir comment elle se superpose à la contrainte sémantique que nous avons mise au jour ici. Précisons néanmoins que cette seconde contrainte, si elle se manifeste certes au passif, en est structurellement indépendante, puisque les propriétés relationnelles liées à la position sujet sont fondées indépendamment du passif.

Concernant le statut du participe passé au passif, il nous faudra également reprendre le débat non tranché à l'heure actuelle : s'agit-il d'un objet linguistique distinct du participe passé à l'actif, ou pas? L'examen des données liées à ces deux classes d'emplois du participe passé nous permettra de préciser la nature de la relation syntaxique et sémantique entre le verbe être et le participe passé au passif et donc de mieux cerner l'identité du passif en français.

\section{BIBLIOGRAPHIE}

Abeillé A. et Godard D. (1996), « La complémentation des auxiliaires français », Langages, 30/122, $32-61$.

Abeillé, A. et Godard, D. (2002), « The syntactic structure of French auxiliaries », Language 78:3, 404-452.

Bolinger, D. (1975), « On the passive in English », in Makkai A. \& Makkai V.B. (éds), The First Lacus Forum, 1974, Columbia S.C. : Hornbeam Press, 57-80.

Bonne, A. (1998), « Essai de typologie des phrases copulatives », in Forsgren et al. (eds.), Prédication, assertion, information, Actes du colloque d'Uppsala en linguistique française, 6-9 juin 1996, Uppsala : Acta Universitatis Upsaliensis, Studia Romanica Upsaliensia, 56, 67-80.

Bouveret M. and Legallois D. (2012), « Cognitive linguistics and the notion of construction in French studies: An Overview ", in Constructions in French, Bouveret M. and Legallois D (eds.), Amsterdam / Philadelphia: John Benjamins Publishing Company, 1-19.

Buchard, A. (2006), « Pour une analyse unitaire de l'attribut du sujet et de l'attribut de l'objet », Travaux de linguistique, 53/2, 67-89.

Buchard, A. (2009), Etre + participe passé » en tant que marqueur d'aspect et de structure argumentale, Thèse, Université de Paris 8.

Carlier, A. (2002), « Les propriétés aspectuelles du passif », Cahiers Chronos, 10, 41-63 ». 
Croft, W. (2001): Radical Construction Grammar: Syntactic theory in typological perspective, Oxford/New York: Oxford University Press.

Culioli, A. ([1971] 1999), « A propos d'opérations intervenant dans le traitement formel des langues naturelles ", Mathématiques et Sciences Humaines, 34, repris dans Pour une linguistique de l'énonciation, T.2, Paris : Ophrys, 31-41.

Culioli, A. ([1982] 1999), « Rôle des représentations métalinguistiques en syntaxe », collection ERA 642, université de Paris VII, repris dans Pour une linguistique de l'énonciation, T.2, Paris : Ophrys, 95-114.

Desclès, J.-P. et Guentcheva Z. (1993), « Le passif dans le système des voix du français », Langages, 109, 73-102.

François, J. (1998), « Théorie multifactorielle de la transitivité, « différentiel de participation » et classes aspectuelles et actancielles de prédication », in André Rousseau (éd.), La transitivité, Villeneuve d'Ascq : Presses Universitaires du Septentrion : 181-201.

Gaatone, D. (1995), « Syntaxe et Sémantique: le cas des verbes transparents », Perspectives, Revue de l'Université Hébraïque de Jérusalem. Le langage et le texte. Hommages A. Lorian, 2, 55-71.

Gaatone, D. (1998), Le passif en français. Paris / Bruxelles : Duculot.

Goldberg, A. (1995), Constructions: A construction grammar approach to argument structure, Chicago : University of Chicago Press.

Goldberg, A. (2013), « Constructionist Approaches to Language », In Thomas Hoffmann and Graeme Trousdale (eds.) Handbook of Construction Grammar, Oxford University Press.

Gosselin, L. (1996), Sémantique de la temporalité en français : un modèle calculatoire et cognitif du temps et de l'aspect, Louvain-La-neuve : Duculot.

Gross M. (1975), Méthodes en syntaxe. Régime des constructions complétives, Paris : Hermann.

Guillet A. ( 1990), « Fondements formels des classes sémantiques dans un lexique-grammaire », Langages, 98, 70-78.

Heine, B. (1993), Auxiliaries. Cognitive Forces and Grammaticalization, New York/Oxford : Oxford university press.

Helland, H. P. (2002), Le passif périphrastique en français moderne. Copenhague : Museum Tusculanum Press.

Herslund M. et al. (1997), Det franske sprog, Capitel IV : Diatese, København, manuscrit.

Hopper, P. et Thompson, S. (1980), « Transitivity in Grammar and Discourse », in Language, 56/2, 251-297.

Jalenques, P. (2010), « Un exemple d'interaction morphologie-syntaxe-sémantique : les contraintes sur les procès transitionnels au passif périphrastique », Actes du VIII ${ }^{\mathrm{e}}$ Congrès International de Linguistique Française, 25-27 septembre 2008, Université d'Oviedo, Liens linguistiques. Etudes sur la combinatoire des composants, Berne : Peter Lang, 409-425.

Jalenques, P. (2010), « Valeurs spatiales et non spatiales du verbe monter : pour une sémantique constructiviste ", Actes des Cinquièmes Rencontres de Sémantique et Pragmatique, Université de Gabès, 22-24 avril 2009, Revue de sémantique et pragmatique, 25-26, pp. 61-80.

Keenan, E. L. (1976), « Towards a Universal Definition of "Subject », in Ch. Li (ed.), Subject and Topic, 303-333.

Kronning, H. (2003), « Auxiliarité, énonciation et rhématicité », Cahiers chronos, 11, 231-249. 
Lambrecht K. (1994) : Information Structure and Sentence Form. Topic, Focus and the Mental Representations of Discourse Referents, Cambridge : Cambridge University Press.

Lamiroy, B. (2000), « Sur certains rapports entre le passif pronominal et le datif », in L. Schösler (éd.), Le Passif, Actes du colloque de Copenhague 5-7 mars 1998, Etudes Romanes, 45, 135-154.

Lamiroy, B. et Melis, L. (2005), « Les copules ressemblent-elles aux auxiliaires ?, in H. Bat-Zeev Shyldkrot \& N. Le Querler (dir.) Les périphrases verbales, Amsterdam/Philadelphia : Benjamins, 145-170.

Lauwers Peter (2009) - La prédication attributive. Portée, structuration interne et statut théorique

Lauwers, P. et Tobback E. (2010), « Les verbes attributifs : inventaire(s) et statut(s) », Langages, 179-180, 79-113.

Lauwers, P. et Melis, L. (2013), «L'attribut du sujet. À la recherche de l'unité dans la diversité », in Aboubakar Ouattara (dir.), Les fonctions grammaticales Histoire, théories, pratiques, GRAMM-R, Études de linguistique française, 18, Peter Lang, 251-262.

Lemaréchal A. (2001), « Mais à quoi peut donc bien servir la copule? », in Buridant et al. (éds.), Par monts et par vaux. Itinéraires linguistiques et grammaticaux, Bibliothèque de l'Information Grammaticale, 45, 235-242.

Leclère, C. (1993), « Classes de constructions directes sans passif », Langages, 109, 7-31.

Martin, R. (1988), « Temporalité et classes de verbes », l'Information grammaticale, 39, 3-8.

Melis, L. (2001), « Les compléments nominaux des verbes de mouvement intransitifs et la constellation de l'objet ». In : C. Buridant et al. (éds), Par monts et par vaux: itinéraires linguistiques et grammaticaux, Louvain: Peeters, 243-258.

Melis, L. (2002), « Objects and quasi-objects: the constellation of the object in French ». In: K. Davidse - B. Lamiroy, The nominative and accusative and their counterparts, Amsterdam: Benjamins, 41-79.

Milner, J.-C. (1986), Introduction à un traitement du passif, coll. ERA 642, Université Paris 7.

Muller, Cl. (2000), « Le passif processif et ses concurrents. Définition et quelques particularités », in L. Schösler (éd.), Le Passif, Actes du colloque de Copenhague 5-7 mars 1998, Etudes Romanes, 45, 49-69.

Perlmutter, D. M. \& Postal P. M. (1977), « Toward a Universal Characterization of Passivization », Proceedings of the 3rd Annual Meeting of the Berkeley Linguistics Society, pp. 394-417.

Raineri, S. (2010), Analyse contrastive français-anglais du passif dans une perspective constructionnelle : sens et fonction de BE Ven, ETRE Vé, GET Ven, SE FAIRE Ver, Thèse, Université Sorbonne Nouvelle - Paris 3.

Riegel, M. (1985), L'adjectif attribut. Paris : Presses Universitaires de France.

Rivière, N. (1990), « Le participe passé est-il verbe ou adjectif ? », Travaux de Linguistique et de Philologie, p. 131-169.

Rouveret, A. (1998), « Points de vue sur le verbe "être” ", in Alain Rouveret (éd.), "être" et "avoir" : syntaxe, sémantique, typologie, Saint-Denis : Presses universitaires de Vincennes, 11-65. Ruwet, N. (1983), « Du bon usage des expressions idiomatiques dans l'argumentation en syntaxe générative », Revue québécoise de linguistique, 13/1, 9-145.

Siewierska, A. (1985), The Passive: A Comparative Linguistic Analysis. London: Croom Helm. 
Sivertsen, I. (1998), « Le verbe de la phrase SN être (X) est la copule », in Forsgren et al. (eds.), Prédication, assertion, information, Actes du colloque d'Uppsala en linguistique française, 6-9 juin 1996, Uppsala : Acta Universitatis Upsaliensis, Studia Romanica Upsaliensia, 56, 523-530.

Van Peteghem, M. (2000), Les phrases copulatives dans les langues romanes, Wilhelmsfeld : Egert.

\section{NOTES}

1. Nous nous abstenons pour l'instant de tout a priori sur la nature de être au passif (auxiliaire ou pas), d'où l'emploi du terme neutre élément.

2. Cf. par exemple Leclere (1993) et Gaatone (1998: 63-115) pour une compilation de nombreux exemples.

3. Nous rejoignons sur ce point les observations faites dans le cadre du lexique-grammaire (cf. Guillet (1990), Leclère (1993).

4. Pour éviter tout malentendu, je précise que j'emploie ici le terme de « construction » dans son sens trivial issu de la tradition grammaticale et non dans le sens technique qu'il a acquis dans le cadre des grammaires de construction (cf. par exemple A. Goldberg, 1995).

5. Un des arguments classiques est le fait que le complément d'agent est généralement facultatif au passif, le caractère facultatif étant une des caractéristiques des compléments non argumentaux.

6. C'est-à-dire un procès dynamique marquant « le passage d'un état à un autre » (Martin, 1988 :

5). Généralement, la notion de procès transitionnel recouvre également les procès de changement de localisation (entrer, sortir). C'est dans ce sens étendu que nous utilisons cette notion.

7. Les séquences en italiques dans les exemples correspondent à des séquences attestées relevées sur internet.

8. Il s'agit bien d'un procès dynamique puisqu'il passe le test avec être en train de identifiant les procès dynamiques (cf. Gosselin (1996)) : Marie était en train de regarder la télé lorsque le téléphone sonna.

9. Indépendamment de la question du passif, Melis (2001) fait des observations similaires.

10. Les emplois correspondant à un procès statif sont considérés comme moins transitifs que les emplois correspondant à un procès dynamique agentif (cf. Hopper et Thompson, 1980).

11. Nous n'entrons pas ici dans le débat polysémie / homonymie concernant cet emploi de monter par rapport aux deux précédents. Que l'on admette pour ces divers emplois un seul verbe monter polysémique (hypothèse que nous défendons ailleurs (cf. Jalenques 2010)), ou bien deux verbes monter homonymes, cela ne change rien au fond de la discussion, à savoir la portée sémantique du procès dans chaque emploi et le constat que le passif contraint cette portée.

12. J'utilise "propriétés notionnelles", simplement comme une étiquette descriptive pour renvoyer au type d'entités («entité» entendu au sens le plus large) désigné par l'argument du verbe, tel que animé humain, moyen de transport, état, qualité, procès statif ou dynamique, etc.

13. Exemples indiqués par François Gaudin (communication personnelle).

14. Raineri (2010: 239) observe à partir d'un corpus de textes de presse que les passifs à sujet en topique sont à peine plus nombreux que les passifs à sujet non topiques.

15. Pour une discussion récente sur les grammaires de construction, je renvoie à Goldberg (2013) ainsi qu'à Bouveret et Legallois (2012).

16. Kronning appelle coverbe " tout verbe qui se construit avec [un complément verbal à] un mode impersonnel - infinitif, participe passé ou participe présent » (p. 232).

17. Rappelons que TAM renvoie à « Temps, Aspect, Modalité ». 
18. Pour une définition précise de l'opposition accompli / inaccompli, je renvoie à Gosselin (1996, chapitre I).

19. De ce point de vue, le participe "passé » porte mal son nom; on voit ici le poids de la terminologie.

20. Une deuxième possibilité de résolution de conflit interprétatif réside dans la construction d'une valeur itérative générique, comme dans le vin est (habituellement) servi par le sommelier; la situation globale prédiquée a elle aussi une valeur aspectuelle stative (pas de changement prédiqué). Voir l'analyse de Carlier pour plus de détails.

21. Cette situation n'a rien d'exceptionnelle. Lamiroy et Melis (2005) montrent également une proximité de fonctionnement entre les semi-auxiliaires et les verbes copules (au sens large).

22. Pour un panorama récent sur la notion d'attribut du sujet, je renvoie à Lauwers et Melis (2013).

23. De même, dans Culioli ([1982] 1999: 114): "passiver, c'est construire une relation doublement converse (changement du terme de départ, changement de l'orientation)».

24. Cf. par exemple Gaatone (1998), C. Muller (2002).

25. En cela, le passif en français se distingue nettement du passif dans bien d'autres langues où l'absence du premier argument du verbe est obligatoire (cf. Croft (2001)).

INDEX

Mots-clés : passif, verbe, idiosyncrasie, copule, polysémie

Keywords : passive construction, verb, idiosyncrasy, copula, polysemy

\section{AUTEUR}

PIERRE JALENQUES

Université de Rouen

pierre.jalenques@univ-rouen.fr 